\title{
Involvement of the Central Thalamus in the Control of Smooth Pursuit Eye Movements
}

\author{
Masaki Tanaka \\ Department of Physiology, Hokkaido University School of Medicine, Sapporo 060-8638, Japan
}

\begin{abstract}
During maintenance of smooth pursuit eye movements, the brain must keep track of pursuit velocity to reconstruct target velocity from motion of retinal images. Although a recent study showed that corollary discharge signals through the thalamus to the cortex are used for internal monitoring of saccades, it remains unknown whether signals in the thalamus also contribute to monitoring and on-line regulation of smooth pursuit. The present study sought possible roles of the thalamocortical pathways in pursuit by recording activities of single thalamic neurons and by analyzing the effects of local inactivation. Data showed that many neurons in the ventrolateral thalamus exhibited directional modulation during pursuit. Most neurons discharged before or during initiation of pursuit, and the firing rate was proportional to the speed of target motion in a preferred direction. When the tracking target was extinguished briefly during maintenance of pursuit, these neurons continued firing, indicating that they carried extra-retinal, eye movement signals. The majority of neurons showed no change in activity around the time of small catch-up saccades during pursuit but responded transiently to large $\left(16^{\circ}\right)$ memory-guided saccades in the preferred pursuit direction. Local inactivation of the recording sites did not alter pursuit latency but reduced eye velocity modestly during initiation and maintenance of ipsiversive pursuit. The results suggest that the central thalamus lies within pathways that regulate and monitor smooth pursuit eye movements.
\end{abstract}

Key words: eye movement; smooth pursuit; central thalamus; primate; single unit; inactivation

\section{Introduction}

Although signals through the thalamus to the cortex are essential for voluntary control of somatic movements, the roles of thalamocortical pathways in the control of eye movements remain primarily unknown. One specific role was demonstrated in a recent study showing that saccade signals in the mediodorsal (MD) thalamus were used to keep track of eye movements and to update subsequent motor plans (Sommer and Wurtz, 2002, $2004 \mathrm{~b}$ ). Internal monitoring of one's own behavior is also an integral part of smooth pursuit, a voluntary eye movement that allows primates to track a small moving object. Previous studies showed that monkeys and humans could update visuospatial working memory during pursuit (Zivotofsky et al., 1996; Baker et al., 2003) and that signals in the thalamus played a role in this updating process, at least in part (Gaymard et al., 1994). Just like saccade signals are used to preserve spatial continuity of visual responses in parietal neurons, pursuit signals also result in remapping of visual receptive fields of those neurons (Powell and

\footnotetext{
Received Feb. 18, 2005; revised May 9, 2005; accepted May 10, 2005.

This work was supported by a grant-in-aid for scientific research on priority areas (Advanced Brain Science Project) and a grant for young scientists from the Ministry of Education, Culture, Sports, Science, and Technology of Japan. I thank J. Gardner for comments on a previous version of this manuscript; S. Hirano and T. Yasuda for technical assistance; M. Takada, M. Watanabe, M. Fukaya, S. Miyachi, and N. Hatanaka for suggestions on histological procedures; and K. Amano for administrative help. I am grateful to M. Takada at the Tokyo Metropolitan Institute for Neuroscience for his help in identifying the thalamic nuclei on histological sections. One monkey was provided by the Primate Research Institute of the Kyoto University by courtesy of Prof. A. Mikami.

Correspondence should be addressed to Masaki Tanaka, Department of Physiology, Hokkaido University School of Medicine, North 15, West 7, Sapporo 060-8638, Japan. E-mail: masaki@med.hokudai.ac.jp.

D0I:10.1523/JNEUROSCI.0676-05.2005

Copyright $\odot 2005$ Society for Neuroscience $\quad$ 0270-6474/05/255866-11\$15.00/0
}

Goldberg, 1997). Furthermore, because the smooth pursuit system operates as a negative feedback system, the system itself must monitor eye velocity continuously to reconstruct target velocity from retinal image motion, which is greatly reduced during maintenance of pursuit (Robinson et al., 1986; Krauzlis and Lisberger, 1994).

Previous studies showed that neurons in the central thalamus carried eye movement signals that were related to saccades, fixation, and eye or gaze position in space (Schlag and Schalg-Rey, 1984, 1986; Tanibuchi and Goldman-Rakic, 2003, 2004; Wyder et al., 2003; Sommer and Wurtz, 2004a; Watanabe and Funahashi, 2004). However, it remains unknown whether the central thalamus also transmits signals that are related to smooth pursuit eye movements. Anatomical data suggest the existence of pursuit signals in the central thalamus. Both the frontal eye field (FEF) and the supplementary eye field that contain pursuit neurons receive abundant projections from the central thalamus, including the rostral group of the intralaminar nuclei and adjacent paralaminar regions of the $\mathrm{MD}$, ventrolateral (VL), and ventroanterior (VA) nucleus of the thalamus (Huerta et al., 1986; Shook et al., 1991; Lynch et al., 1994; Matelli and Luppino, 1996; Tian and Lynch, 1997). Conversely, the central thalamus receives inputs from subcortical pursuit pathways, including the vestibular nuclei (Lang et al., 1979; Asanuma et al., 1983) and the deep cerebellar nuclei (Kalil, 1981; Asanuma et al., 1983; Lynch et al., 1994).

The present study aimed to elucidate the possible roles of the central thalamus in the control and monitoring of smooth pursuit by recording activity of single thalamic neurons and by examining effects of local inactivation. The results showed that many neurons in the ventrolateral thalamus were active during 
pursuit, and inactivation of these neurons reduced eye velocity during initiation and maintenance of pursuit, suggesting that the central thalamus plays roles in smooth pursuit control.

Parts of the this work have been presented previously in abstract form (Tanaka, 2004).

\section{Materials and Methods}

Animal preparation. Three adult Japanese monkeys (Macaca fuscata; one male, two females, 6-12 kg) were used. Two of them participated in previous behavioral experiments (Tanaka, 2003). All experimental protocols described here were approved in advance by the Animal Care and Use Committee of the Hokkaido University School of Medicine and were in accordance with the Guide for the Care and Use of Laboratory Animals (National Research Council, 1996). After the animals were trained to sit in a primate chair, a pair of head holders was implanted to the skull using titanium screws and dental acrylic under general halothane and pentobarbital anesthesia and using sterile procedures. A few weeks after the first surgery, a coil of stainless steel wire was implanted under the conjunctiva to record eye movements, using the same surgical procedures. During subsequent training and experimental sessions, the monkey's head was secured to the primate chair, and horizontal and vertical eye position were recorded by using the search coil technique (Fuchs and Robinson, 1966). After training with the oculomotor tasks and behavioral experiments that lasted for several months, a recording cylinder (19 $\mathrm{mm}$ in diameter) was installed over a small craniotomy that was centered at A10-A13, L5, under the same surgical conditions. To verify the location of the thalamus, magnetic resonance images were taken from two monkeys before surgery. Animals received analgesia with either suppository acetaminophen or intramuscular injection of pentazocine after each surgery. Topical antibiotics were administered around the implant and in the cylinder as necessary. Water intake of monkeys was controlled daily so that they were motivated to perform oculomotor tasks.

Visual stimulus and behavioral paradigms. Experiments were controlled by a Windows-based real-time data acquisition system (TEMPO; Reflective Computing, St. Louis, MO) running on Pentium personal computers. All events were updated every $5 \mathrm{~ms}$, and visual stimuli were presented on a 24-inch cathode-ray tube monitor (GDM-FW900, refresh rate $60 \mathrm{~Hz}$; Sony, Tokyo, Japan) that was located $38 \mathrm{~cm}$ away from the eyes and subtended $64 \times 44^{\circ}$ of visual angle. A $0.5^{\circ}$ square spot served as the visual stimulus. Experiments were performed in a darkened booth, but visual stimuli were presented on a gray background $\left(0.05 \mathrm{~cd} / \mathrm{m}^{2}\right)$ to reduce visible streaks of a moving target. Horizontal and vertical eye position signals were calibrated before each experiment by having monkeys fixate a stationary target spot at known visual angles. Thereafter, visual stimuli were presented in individual trials, and monkeys were reinforced with drops of water or apple juice for maintaining eye position within a "window" that surrounded the target position throughout each trial. The trial was aborted and followed by newly selected trials if monkeys failed to maintain eye position within a specified window.

Each trial began with the onset of a red fixation target $\left(2.0 \mathrm{~cd} / \mathrm{m}^{2}\right)$. In the step-ramp pursuit task, the target was extinguished after a random $800-1200 \mathrm{~ms}$ interval, and a white moving target $\left(2.3 \mathrm{~cd} / \mathrm{m}^{2}\right)$ appeared $1-8^{\circ}$ from the location of the initial fixation. The target moved toward the fixation location at $5,10,20,30$, or $40 \%$ s so that it crossed the fixation location $200 \mathrm{~ms}$ after motion onset. Location of initial fixation was at the center of the screen with a target speed of $5-20^{\circ}$ s but was shifted by $10^{\circ}$ for faster target speeds. After a motion period of $900 \mathrm{~ms}$ for a target speed of $40 \% \mathrm{~s}$ and $1000 \mathrm{~ms}$ for other speeds, the target stopped and remained visible for an additional $900-1300 \mathrm{~ms}$. Monkeys were required to move their eyes within a $2-4^{\circ}$ window surrounding the target location throughout the trial except for the initial $300 \mathrm{~ms}$ interval of target motion. In a variant of the pursuit task, the tracking target was extinguished briefly (150 ms) $250 \mathrm{~ms}$ after motion onset to examine the contribution of the retinal images of the target spot to the smooth pursuit-related neuronal activity. In the memory-guided saccade task, a white target spot appeared at $16^{\circ}$ peripherally for $200 \mathrm{~ms}$ during a central fixation of $800-$ $1200 \mathrm{~ms}$. Animals were required to withhold responding to the target and maintained fixation at the center of the screen for another second. After offset of the fixation point, monkeys were required to make a saccade to the location of the previously flashed target within $400 \mathrm{~ms}$. The peripheral target reappeared at the same location $400 \mathrm{~ms}$ after offset of the fixation point and was visible for an additional $800-1200 \mathrm{~ms}$ until the end of the trial. The second fixation interval was introduced to examine eye position-related modulation during fixation.

Procedures for recording and microinjection. A tungsten microelectrode (4-7 M $\Omega$ at $1 \mathrm{kHz}$; Frederick Haer Company, Bowdoinham, ME) was lowered through a 23 gauge guide tube with a hydraulic micromanipulator (MO-97S; Narishige, Tokyo, Japan). At the beginning of each experimental session, the location of electrode penetration was adjusted by using an $x-y$ stage attached on top of the cylinder, and the guide tube was advanced 10-15 $\mathrm{mm}$ from the surface of intact dura. Signals through the electrodes were amplified, filtered, and monitored by using oscilloscopes and an audiomonitor. Typically, the dorsal surface of the thalamus could be identified by the existence of active fibers followed by the lateral ventricle. When neuronal activity related to eye movements was searched for, monkeys performed a block of memory-guided saccade trials $\left(16^{\circ}\right)$ and pursuit trials $(20 \%)$ in four cardinal directions or in four oblique directions. Once task-related neuronal activity was encountered, isolation of single neurons was attempted by using a real-time spike sorter with template-matching algorithm (MSD; Alpha Omega, Nazareth Illit, Israel). Optimal direction of each task-related neuron was determined by changing the direction of the target motion in $45^{\circ}$ steps, and neuronal activity was examined for eye movements along the preferred axis. Occurrence of action potentials of single neurons was detected and was saved in files with the data of eye movements and the location and timing of visual stimuli during the experiments.

After the recording experiments were terminated, effects of local inactivation of the central thalamus were examined in separate experiments. A 30 gauge injection needle connected to a microsyringe was inserted through the guide tube, and a small amount of GABA agonist (muscimol hydrobromide; $5.0 \mu \mathrm{g} / \mu \mathrm{l}$ in saline, $1.0-2.0 \mu \mathrm{l}$ ) was pressure injected around the recording sites at a rate of $\sim 0.2 \mu \mathrm{l} / 30 \mathrm{~s}$. The effect of inactivation was assessed by comparing pursuit velocity before and $10-90 \mathrm{~min}$ after injection. A previous study showed that the spread of muscimol ( $1 \mu \mathrm{g}$ in $1 \mu \mathrm{l})$ injected into the rat cerebral cortex was $\sim 1.7 \mathrm{~mm}$, and that the width of spread remained relatively constant for 30-120 min after injection (Martin, 1991).

Data acquisition and analysis. Horizontal and vertical eye position signals were obtained directly from the eye coil electronics (MEL-25; Enzanshi Kogyo, Tokyo, Japan). Signals proportional to horizontal and vertical eye velocity were obtained by passing voltages of eye position signals through analog circuits that differentiated frequencies up to 50 $\mathrm{Hz}$ and attenuated higher frequencies $(-12 \mathrm{~dB} /$ octave). Eye position, eye velocity, and target position signals were digitized at $1 \mathrm{kHz}$ and were stored in files with other codes of task events. Times of action potentials and the onset of each vertical retrace were also stored in the same files for subsequent off-line analysis that was performed using Matlab (MathWorks, Natick, MA).

For each neuron, traces of horizontal and vertical eye position were reviewed with the rasters and spike density profile that were constructed from the neuronal data. To obtain spike density, means of the millisecond-by-millisecond occurrence of action potentials across multiple trials were convolved with a Gaussian kernel that had a $\sigma$ of either 15 or $10 \mathrm{~ms}$. The $15 \mathrm{~ms}$ kernel was used to show time courses of individual neuronal activities, whereas the $10 \mathrm{~ms}$ kernel was used to construct the population activity, to measure the latency of pursuit-related neuronal activity and to determine whether each pursuit neuron showed changes in activity around the time of catch-up saccades. Other quantitative measurements of neuronal activity were made based on spike counts for specific time windows.

When pursuit and neuronal latencies were measured, data were aligned on the exact time of target motion onset that was computed based on target location and vertical retrace signals (Tanaka, 2003). To determine the onset of pursuit-related activity, spike density after target motion was compared with the variance of average spike density over the 400 ms period immediately before target motion (baseline). The onset of neuronal activity corresponded to the beginning of the first $50 \mathrm{~ms}$ interval when average spike density consistently exceeded the mean +2 SDs 
Table 1. Number of neurons with different types of task-related activity

\begin{tabular}{lc}
\hline Smooth pursuit & $55(21 \%)$ \\
\hline Eye position & $40(15 \%)$ \\
All saccade & $181(70 \%)$ \\
Saccade with pursuit & 45 \\
All delay & $51(20 \%)$ \\
Delay with pursuit & 17 \\
Delay with saccade & 30 \\
Delay with both & 11 \\
Others & $21(8 \%)$ \\
Total & 260 \\
\hline
\end{tabular}

Because many neurons showed task-related activity for more than one task event, the sum of the numbers of neurons exceeds the total number of neurons.

of the baseline activity. To measure pursuit latency, the millisecond-bymillisecond distribution of desaccaded eye velocity was compared with the distribution of baseline eye velocity $(100 \mathrm{~ms}$ before target motion onset) by using an unpaired $t$ test. The beginning of the first $100 \mathrm{~ms}$ period when the $p$ value was consistently $<0.01$ was taken as the pursuit onset (see Figs. 4, 13). Similarly, latency of the effects of thalamic inactivation on pursuit was measured by comparing the millisecond-bymillisecond distributions of eye velocity before and after inactivation, and the beginning of the first $100 \mathrm{~ms}$ period when eye velocity was consistently different ( $t$ test, $p<0.01$ ) was taken as the onset of the inactivation effects (see Fig. 13).

Directionality of pursuit-related activity was quantified by computing the directional index (DI) that was defined as, $D I=1-$ Opp/Pref, where Pref was the modulation of neuronal activity for trials in the preferred direction, and Opp was that measured for the same time interval but for trials in the opposite direction (see Fig. 3B). Neuronal modulation during pursuit was the difference in activity between a $900 \mathrm{~ms}$ interval starting from $100 \mathrm{~ms}$ after target motion onset to the time of target motion offset, and the baseline activity that was measured for the $400 \mathrm{~ms}$ interval immediately before target motion. The DI was more than a value of 1 when activity was suppressed during pursuit in the direction opposite to the preferred direction. Directionality was also assessed by performing a statistical test (Wilcoxon's rank-sum test) between trials in opposite directions.

Histological procedures. A postmortem examination of the recording sites was performed for one monkey. At the end of the experiments, several electrolytic lesions were made by passing $10-25 \mu$ A negative current through the recording electrodes for $30-40 \mathrm{~s}(600-1000 \mu \mathrm{C})$. The monkey was deeply anesthetized with a lethal dose of pentobarbital $(>50$ $\mathrm{mg} / \mathrm{kg}$ ) and was perfused transcardially with $0.1 \mathrm{~m}$ phosphate buffer, followed by $10 \%$ Formalin with $5 \%$ picrotoxine. Then, the brain was removed, blocked, and fixed with the same solution overnight. Once the brain was equilibrated with $0.1 \mathrm{M}$ phosphate buffer containing $30 \%$ sucrose, $50-\mu \mathrm{m}$-thick coronal sections were cut using a freezing microtome. To verify the locations of intralaminar nuclei of the thalamus, every fifth section was immunohistochemically stained using the mouse monoclonal antibody against acetylcholinesterase (MAB337; Chemicon, Temecula, CA). The remaining sections were stained with cresyl violet.

\section{Results}

Types of neuronal responses and their locations within the thalamus

Data collected from four thalami of three monkeys showed neuronal activity related to smooth pursuit, eye position, saccades, and delay period in the memory-guided saccade tasks. Because response properties during saccades and fixation have been described previously (Schlag and Schlag-Rey, 1984; Schlag-Rey and Schlag, 1984; Tanibuchi and Goldman-Rakic, 2003, 2005; Wyder et al., 2003, 2004; Sommer and Wurtz 2004a; Watanabe and Funahashi, 2004), the present study only considered activity during smooth pursuit. However, to show the overall distribution of eye movement-related neurons in the central thalamus, Table 1 summarizes the number of all task-related neurons that were exam-
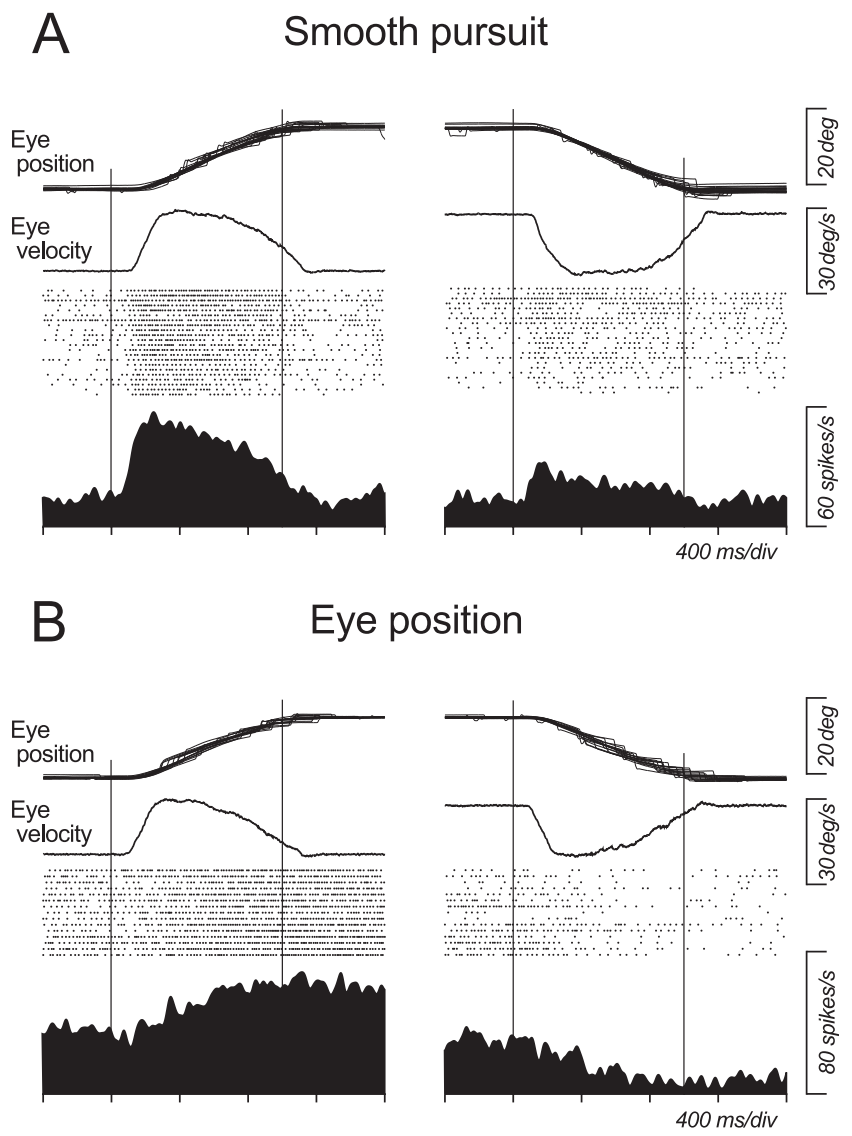

Figure 1. Comparison between pursuit-related and eye position-related activities during smooth pursuit. $\boldsymbol{A}$, Pursuit-related neuron. $\boldsymbol{B}$, Eye position-related neuron. Both neurons show greater activity for ipsiversive than contraversive pursuit. All trials presented here began with central fixation, but traces of eye position and average eye velocity are shifted vertically for presentation purpose only. Vertical lines indicate onset and offset of target motion, respectively.

ined in this study. Activity in almost all of these neurons (259 of 260) was formally examined during pursuit, and 55 (21\%) of them exhibited pursuit-related responses. Activity of 40 other neurons was also modulated during pursuit, but firing rate was related to orbital eye position. Figure 1 shows representative examples of two types of neurons that showed sustained activity during ipsiversive pursuit. Although both neurons modulated their activity depending on the direction of pursuit, the activity during the second fixation interval that was introduced after target motion clearly differentiated these two types of neurons. The pursuit-related neuron (Fig. $1 \mathrm{~A}$ ) ceased firing after target motion offset, whereas the eye position-related neuron (Fig. $1 B$ ) continued firing as long as the animal looked into the preferred direction. As shown in a later section, many pursuit-related neurons also responded only transiently to large memory-guided saccades in the preferred pursuit direction, whereas the eye positionrelated neuron showed sustained activity immediately before or after saccades.

Figure 2 illustrates the sites of neurons recorded from one monkey. To allow for comparisons with other types of neurons, locations of all types of neurons are shown. Pursuit-related neurons distributed rather sparsely in the medial part of the caudal division of the ventrolateral nucleus and adjacent area $\mathrm{X}$ of the thalamus. These neurons were found within an area containing other types of neurons and were close to the area in which previous experiments recorded eye movement-related neu- 

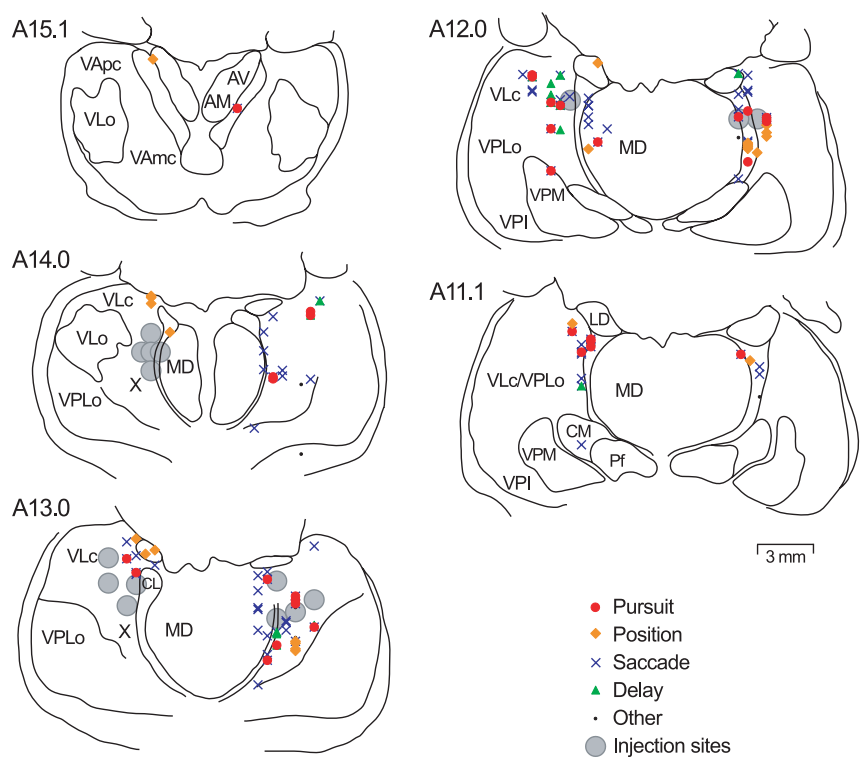

- Pursuit

- Position

$\times$ Saccade

- Delay

- Other

Injection sites

Figure 2. Sites of task-related neurons and inactivation for one monkey. Different symbols are used for different neuronal responses. For many neurons, several symbols are plotted at the same location because those neurons show activity for more than one task event. AM, Anteromedial; $\mathrm{AV}$, anteroventral; $\mathrm{CL}$, centrolateral; $\mathrm{CM}$, center médian; LD, laterodorsal; $\mathrm{MD}$, mediodorsal; Pf, parafascicular; VApc, ventroanterior, parvicellular division; VAmc, ventroanterior, magnocellular division; VLc, ventrolateral, caudal division; VLo, ventrolateral, oral division; VPI, ventroposterioinferior; VPLo, ventroposterolateral, oral division; VPM, ventroposteromedial; $X$, area X.
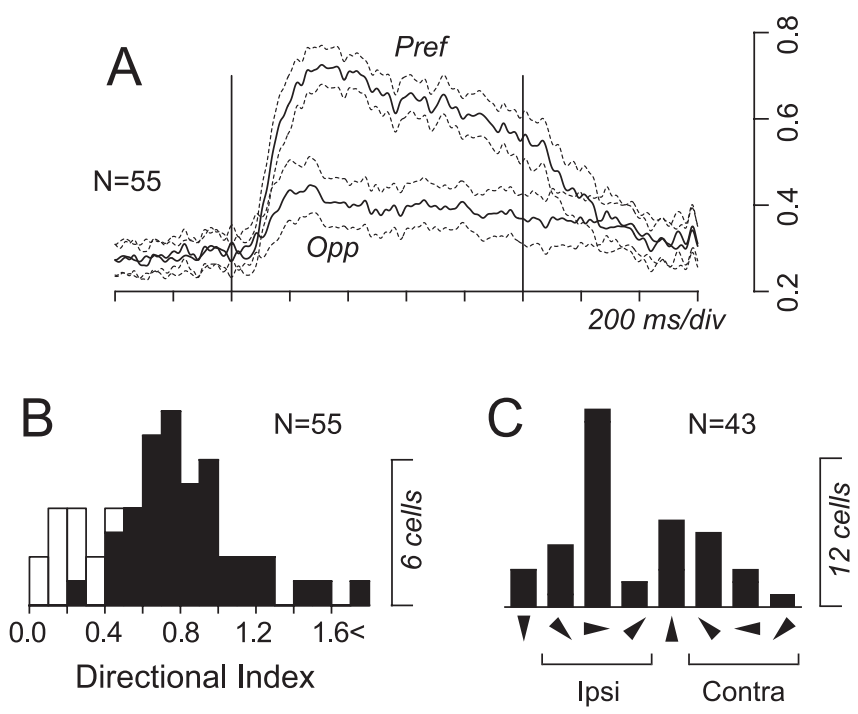

Figure 3. Directionality of pursuit-related activity. $A$, Time course of population activity during pursuit in the preferred (Pref) and opposite (Opp) directions. Broken traces indicate $95 \%$ confidence intervals. Vertical lines indicate onset and offset of target motion at $20 \%$ s. B, Distribution of directional index (see Materials and Methods). Data for neurons that showed statistically significant directional modulation during pursuit $(p<0.05)$ are shown as filled bars. $\boldsymbol{C}$, Distribution of preferred directions relative to the recording side.

rons (Schlag-Rey and Schlag, 1984; Wyder et al., 2003, 2004; Tanibuchi and Goldman-Rakic, 2005).

\section{Directionality of pursuit-related neuronal activity}

Similarly to the neuron shown in Figure $1 A$, most pursuit-related neurons exhibited directional modulation during pursuit. Figure $3 A$ plots the time courses of population activity during pursuit at $20 \% \mathrm{~s}$ in the preferred direction and in the opposite direction. To construct these traces, spike density curves $(\sigma=10 \mathrm{~ms})$ for each

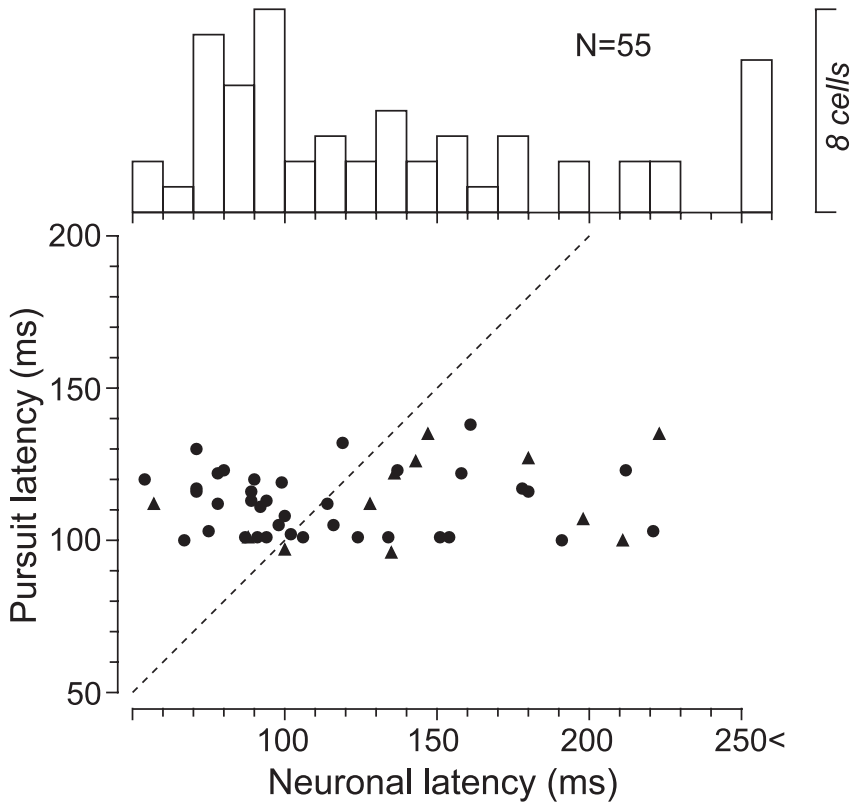

Figure 4. Comparisons between pursuit and neuronal latencies. Data for six neurons that showed latency longer than $250 \mathrm{~ms}$ (ranging from 286 to $317 \mathrm{~ms}$ ) have been omitted from the scatter plot but contribute to the top histogram. Triangles indicate data for neurons that showed modulation of activity around the time of catch-up saccades.

neuron were normalized for their maximal value. Traces in Figure $3 A$ show that activity during pursuit was directional in the population, but there were some responses for pursuit in the opposite direction. Directionality of individual neurons was assessed by computing the directional index (see Materials and Methods) that is plotted in Figure 3B. Distribution of DIs indicates that most neurons showed directional modulation during pursuit, whereas a few responded equally to pursuit in opposite directions. The DIs averaged $0.70 \pm 0.39$ (median, 0.70), and activity during pursuit in opposite directions was statistically different (Wilcoxon's rank-sum test, $p<0.05$ ) for 43 of 55 neurons (78\%) (Fig. 3B, filled bars). For these 43 neurons, Figure $3 C$ plots the distribution of their preferred directions relative to the recording site. The preferred directions were distributed in all directions, including oblique directions, but were biased toward ipsiversive pursuit (Rayleigh's test, $p<0.05$; mean vector angle, $22.6^{\circ}$ ). The width of directional tuning was examined for 23 neurons by having monkeys track a moving target in eight different directions. For 17 of these neurons, average firing rate during target motion (1000 ms in duration) significantly correlated with direction of motion (Mardia's rank correlation, $p<0.05$ ). When a Gaussian curve was fitted to the means of neuronal activity for eight pursuit directions for each of the 17 neurons (least squares, minimal $\left.r^{2}=0.74\right)$, the $\sigma$ of Gaussian averaged $67.0 \pm 22.7^{\circ}$ (median, $67.1^{\circ}$ ), which corresponded to the full-width at halfmaximum of $157.8 \pm 53.4^{\circ}$.

\section{Neuronal response latency}

Figure 4 compares neuronal and pursuit latencies for each pursuit-related neuron. Pursuit latency is the time when eye velocity is statistically different ( $p<0.01$, two-tailed $t$ test) from baseline eye velocity ( $100 \mathrm{~ms}$ before target motion onset) for $>100 \mathrm{~ms}$ (see Materials and Methods). Neuronal latency was measured when the spike density ( $\sigma=10 \mathrm{~ms})$ exceeded the mean +2 SDs of baseline activity ( $400 \mathrm{~ms}$ before target motion onset) for $>50$ ms. The scatter plot in Figure 4 shows that many pursuit-related 
neurons discharged before $(42 \% ; n=23)$ or during initiation of pursuit, and most of them $(82 \% ; n=45)$ were active before the tracking target crossed the initial fixation location $(200 \mathrm{~ms}$ after target motion onset). Neuronal and behavioral latencies averaged $141.5 \pm 71.5 \mathrm{~ms}$ (median, $119 \mathrm{~ms}$ ) and $112.1 \pm 11.5 \mathrm{~ms}$ (median, $112 \mathrm{~ms}$ ) after target motion onset, respectively. On average, neuronal activity lagged pursuit initiation by $29.4 \pm 72.9 \mathrm{~ms}$ (median, $11 \mathrm{~ms}$ ). After removing six outliers with a neuronal latency $>250 \mathrm{~ms}$, these values were $9.2 \pm 46.2 \mathrm{~ms}$ (median, $2 \mathrm{~ms}$ ) with a firing rate lagging pursuit. To confirm that latency of neuronal activity was constant for different tracking conditions, neuronal latency was compared across pursuit for different target speeds in the preferred direction. For 16 neurons that were also analyzed in the next section, neuronal latency for target motion of 5, 10, 20, 30 , and $40 \%$ s averaged $182.1 \pm 107.4$ (median, 141 ), $139.6 \pm 39.4$ (137), $134.5 \pm 53.8$ (131), $144.3 \pm 52.6$ (146), and $142.9 \pm 48.3$ (132.5) ms, respectively, and these values were not statistically different across different target speeds (one-way ANOVA, $p=$ 0.27 ). As described below (see Response to saccades), a minority of neurons $(n=13 ; 24 \%)$ showed changes in activity around the time of catch-up saccades. Neuronal latency of these neurons (Fig. 4, triangles) was not statistically different from that of the others (unpaired $t$ test, $p=0.39$ ).

\section{Velocity sensitivity}

Pursuit-related activity was sensitive to ongoing pursuit velocity. Sensitivity to the target speed was examined by having monkeys track a target that moved at $5,10,20,30$, and $40 \%$ s in the preferred direction (Fig. 5A). Because pursuit-related neurons were insensitive to eye position, as shown in the population activity (Fig. 3A) and as analyzed below, initial fixation location was shifted by $10^{\circ}$ for faster target motion so that duration of target motion was 900 $\mathrm{ms}$ for $40 \%$ s and was $1000 \mathrm{~ms}$ for other target speeds. Figure $5 B$ shows data from a neuron that had a preference for ipsidownward pursuit. Activity of this neuron became greater with increasing speed of target motion. Activity during pursuit was measured for the interval starting at $100 \mathrm{~ms}$ after target motion onset and ending at the time of target stop and is plotted in Figure $5 C$ as a function of target speed. Horizontal lines in the plot indicate means of activity during fixation that were measured for $300 \mathrm{~ms}$ intervals immediately before target motion onset (continuous line) or $300 \mathrm{~ms}$ after target motion offset (broken line), showing that the neuron lacked eye position sensitivity. Figure $5 D$ summarizes the response during pursuit for different target speeds for individual neurons (thin lines) and for population means (thick solid lines). For all neurons tested, magnitude of response increased with increasing speed of target motion. Sensitivity to target speed in the preferred direction was computed by fitting a regression line to data of five target speeds. Regression slopes averaged $0.49 \pm 0.32$ (median, 0.50$)$ spikes $/ \mathrm{s} / \%$, and regression coefficients averaged $0.79 \pm 0.37$

\section{Response in the absence of a tracking target}

Pursuit-related signals in the central thalamus appear to have a nonvisual origin. Contribution of motion of the retinal target images to activity during pursuit was examined by extinguishing the tracking target briefly $(150 \mathrm{~ms})$. Figure $6 A$ illustrates the data of a single pursuit-related neuron that showed preference for ipsiversive pursuit. In half of the pursuit trials at $20 \%$ in the preferred direction, the target was turned off $250 \mathrm{~ms}$ after its motion onset ("Blink"). Rasters and spike density profile for the trials with target blink showed that the neuron decreased its activity only slightly after target blink but continued firing well
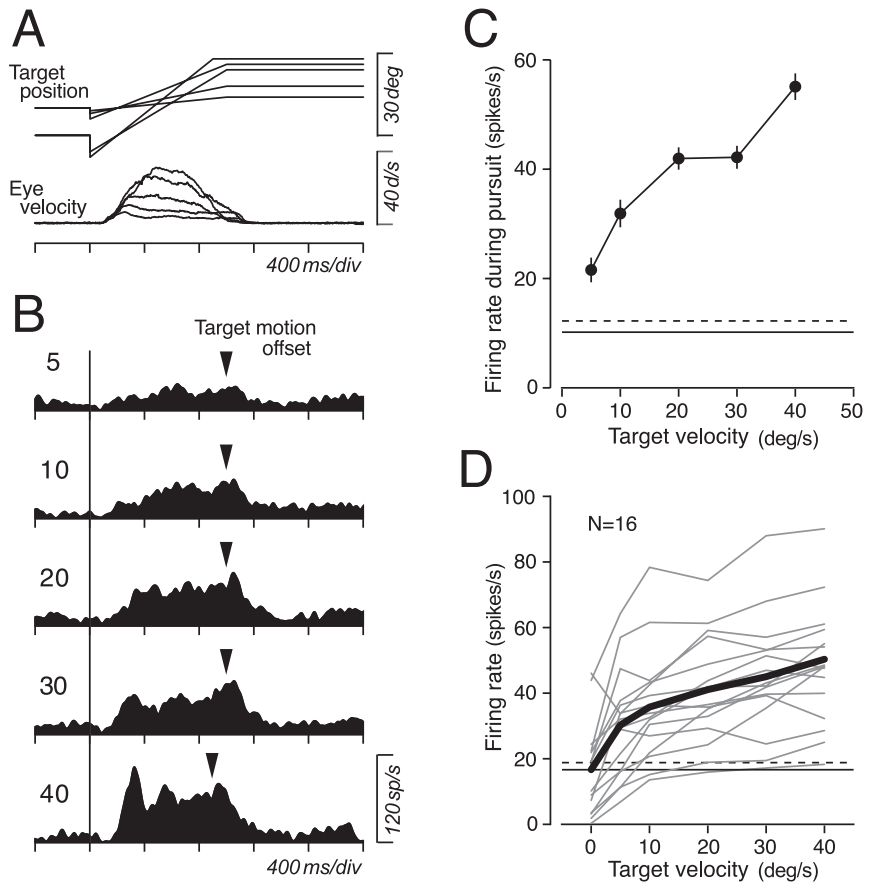

Figure 5. Target velocity sensitivity of pursuit-related activity. $\boldsymbol{A}$, Series of target trajectories used to examine velocity sensitivity and averages of desaccaded eye velocity for the neuron shown in $\boldsymbol{B}$. $\boldsymbol{B}$, Responses of an example neuron. Numbers indicate target speed in the preferred direction. Triangles indicate offset of target motion. $\boldsymbol{C}$, Means \pm SE of neuronal response as a function of target velocity for the neuron shown in $\boldsymbol{B}$. Horizontal lines indicate the mean firing rate during fixation before (solid line) and after (broken line) target motion. D, Quantitative data for this and 15 additional pursuit-related neurons. Data connected with thin lines indicate individual neurons, and those connected with thick solid lines indicate means of the population. Data at zero velocity indicate activity before target motion onset. Horizontal lines indicate means of activity before and after target motion.

above the baseline activity before target motion. The effect of target blink on pursuit-related activity was quantified by measuring the activity for a $100 \mathrm{~ms}$ interval starting from $100 \mathrm{~ms}$ after offset of the tracking target. Figure $6 \mathrm{~B}$ compares means of neuronal activity measured for the trials with target blink and those without it, for 15 thalamic neurons. Except for four neurons that showed statistically significant changes in activity during target blink (filled symbols) (unpaired $t$ test, $p<0.05$ ), activity of most neurons was not altered. The ratio of the magnitudes of activity for trials with to those without target blink averaged $0.98 \pm 0.59$ (median, 0.93).

\section{Response to saccades}

Previous studies in the brainstem have shown that pursuit signals interacted with saccade signals in individual neurons. For example, neurons in the superior colliculus carried motor error signals that were used for control of both pursuit and saccades (Krauzlis et al., 1997). Other studies showed interactions between pursuit and saccade signals for single neurons in the vestibular nuclei (Chubb and Fuchs, 1982; Lisberger et al., 1994), the fastigial nucleus (Fuchs et al., 1994), the interstitial nucleus of Cajal (Missal et al., 2000), and the pontine reticular formation (Keller and Missal, 2003). Because neurons in all of these structures send axons to the paralaminar regions of the thalamus, central thalamic neurons likely respond to both pursuit and saccades. Indeed, most (45 of 55) of the pursuit-related neurons showed a modulation in activity during saccades in the preferred pursuit direction. Figure 7 illustrates three such examples in which all 


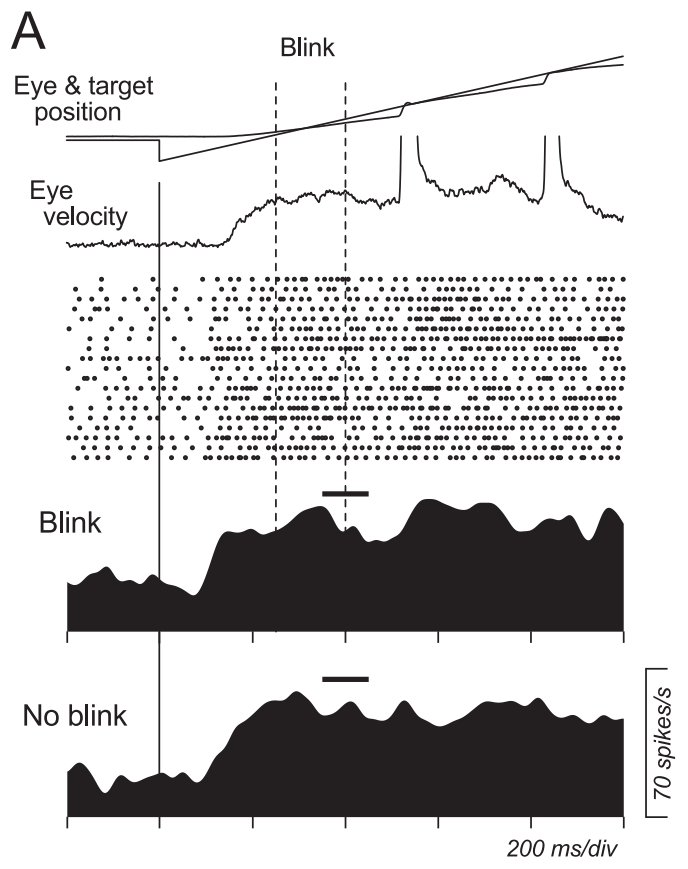

Figure 6. Responses to target "blink" during maintenance of pursuit. $\boldsymbol{A}$, Data for an example neuron. In one-half of the trials, the moving target disappeared only briefly $(150 \mathrm{~ms})$ during pursuit. The raster display is shown for trials with target blink. Horizontal black bars indicate the $100 \mathrm{~ms}$ interval for the quantitative analysis. $\boldsymbol{B}$, Comparison of responses during target blink with nonblink control. Filled circles are data for neurons that showed significant changes in activity during target blink ( $t$ test, $p<0.05$ ).

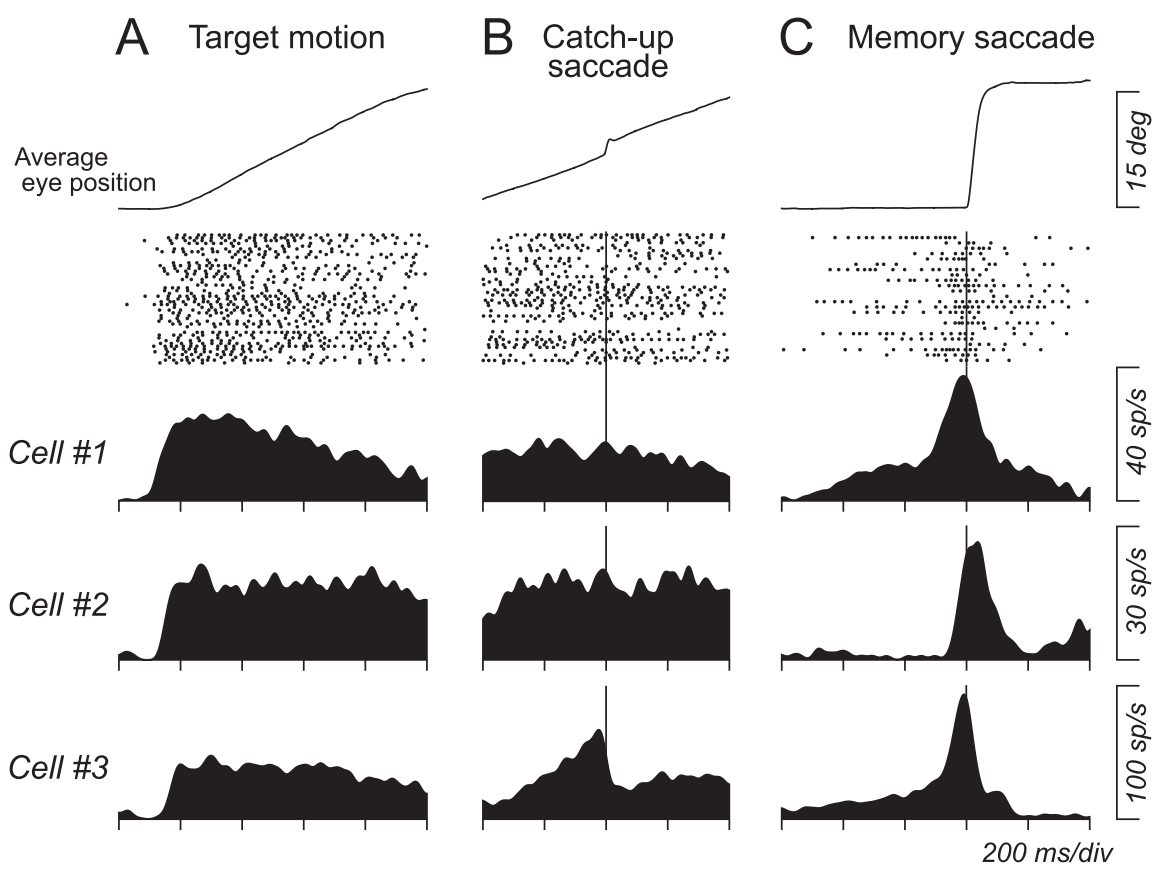

Figure 7. Comparisons between responses to pursuit, catch-up saccades, and large $\left(16^{\circ}\right)$ memory-guided saccades in the preferred pursuit direction for three example neurons. A, Activity during pursuit in the preferred direction. Data are shown for a $1 \mathrm{~s}$ interval of target motion. Average eye position and rasters are shown for cell 1 only. $\boldsymbol{B}$, Same data as in $\boldsymbol{A}$ but aligned on catch-up saccades that occurred $200-800$ ms after target motion onset. $\boldsymbol{C}$, Responses during memory-guided saccades in the preferred pursuit direction.

neurons exhibited sustained activity during pursuit in the preferred direction $(A)$ and also responded to large $\left(16^{\circ}\right)$ memoryguided saccades in the same direction $(C)$. Burst of activity was preceded by a variable amount of buildup of activity during the delay between the cue and fixation target offset. Because these neurons modulated their activity for saccades in the preferred pursuit direction, activity during pursuit could be attributed to occurrence of or preparation for catch-up saccades. If this were the case, activity during pursuit would be modulated around the time of catch-up saccades. To examine this possibility, data for pursuit trials were aligned on catch-up saccades that occurred $200-$ $800 \mathrm{~ms}$ after target motion onset. Then response to catch-up saccades was evaluated whether the mean spike density $(\sigma=10$ $\mathrm{ms}$ ) around the time of catch-up saccade $( \pm 200 \mathrm{~ms})$ deviated from the mean \pm 2 SD of the baseline activity for $>15 \mathrm{~ms}$ that was measured for spike densities of individual saccades and for the interval $200-$ $400 \mathrm{~ms}$ before initiation of saccades. Among 55 neurons, $42(76 \%)$ did not fulfill the above criterion and showed no evident changes in activity around the time of catch-up saccades, as shown by the two examples in Figure 7 (cells 1,2). The remaining 13 neurons showed a gradual increase in activity before initiation of catch-up saccades, just like cell 3 in Figure 7.

Figure $8, A$ and $B$, plots time courses of population activity for both types of neurons that are aligned on the occurrence of catch-up saccades during pursuit in the preferred direction (left column) or on the initiation of memory-guided saccades of $16^{\circ}$ in the preferred pursuit direction (right column, Pref) and in the opposite direction (Opp). To obtain population activity, the spike density ( $\sigma=10 \mathrm{~ms})$ for each neuron was normalized for the value that was used to normalize the pursuitrelated activity shown in Figure $3 A$. For most neurons $(76 \%)$, firing rate was unchanged in the wake of catch-up saccades, but the same neurons modulated their activity around the time of large memoryguided saccades in the preferred or in the opposite direction (Fig. $8 \mathrm{~A}$, right). A minority of neurons (24\%) showed a gradual increase in activity before catch-up saccades and a decrease in activity immediately after saccades (Fig. $8 \mathrm{~B}$, left). The difference in activity between catch-up saccades and large memory-guided saccades could be attributed to the difference in saccade amplitudes. To test this possibility, activity of 11 pursuit-related neurons that showed no clear changes in activity around the time of catch-up saccades was examined for small visually guided saccades in the preferred pursuit direction. Data for the target steps of 1,2 , and $4^{\circ}$ were combined and were aligned with saccade initiation. Neuronal response was computed as the difference between the maximal value of the means of firing rate measured for every $80 \mathrm{~ms}$ intervals around the time of saccades (200 ms before to $400 \mathrm{~ms}$ after saccade initiation) and the activity 300-200 ms before saccades (baseline). For compar- 


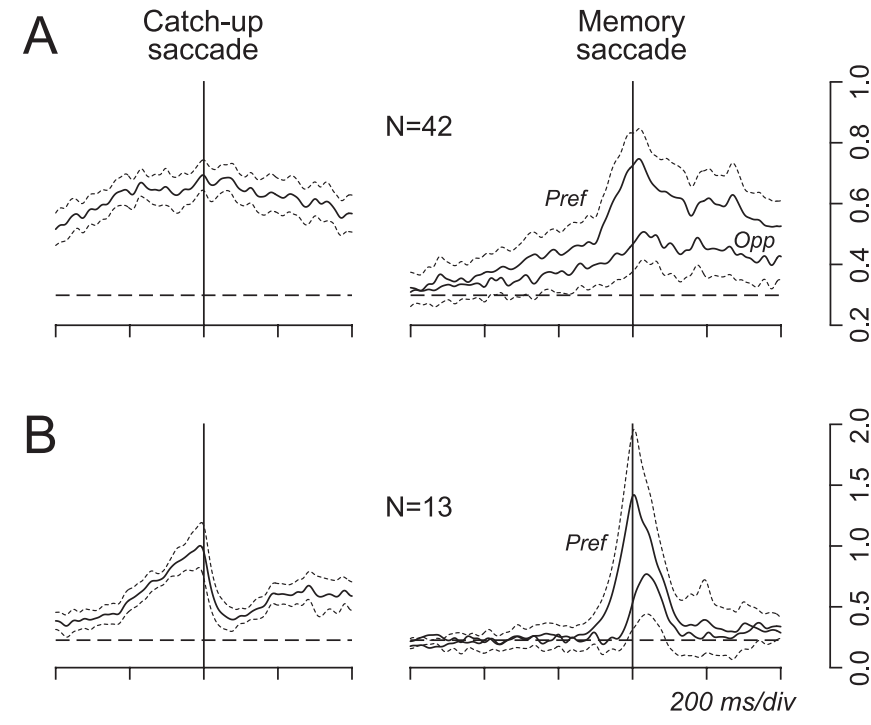

Figure 8. Population activity aligned on catch-up saccades or memory-guided saccades of $16^{\circ}$ in the preferred pursuit direction. $\boldsymbol{A}$, Data for 42 neurons that showed no changes in activity during catch-up saccades. Spike density ( $\sigma=10 \mathrm{~ms}$ ) for each neuron was normalized for the maximum value of the response during pursuit aligned on target motion onset, as in Figure $3 A$. Continuous traces on the right show responses for memory-guided saccades in both directions. Thin broken traces indicate $95 \%$ confidence intervals. Horizontal broken lines indicate baseline activity measured for $400 \mathrm{~ms}$ before target motion onset. $\boldsymbol{B}$, Data for 13 neurons that showed a change in activity around the time of catch-up saccades. Pref, Preferred direction; 0pp, opposite direction.

ison, response to catch-up saccades was also measured for the same intervals, and response to large memory-guided saccades was measured by using a $150 \mathrm{~ms}$ time window. When neuronal responses to small saccades were normalized for response to large memory-guided saccades, the values averaged $0.49 \pm 0.29(n=$ 11 ) and $0.02 \pm 0.35$ for visually guided saccades and catch-up saccades, respectively. A one-way ANOVA and post hoc comparison (Scheffe's) revealed that firing rates for catch-up saccades were statistically different from those during amplitude-matched visually guided saccades $(p<0.05)$, and that firing rates for large memory-guided saccades were different from those during small saccades $(p<0.01)$. Thus, absence of activity modulation around the time of catch-up saccades appeared to be attributable to both the facts that they were small saccades and that they were generated during pursuit.

To compare the activity during pursuit with that around the time of large saccades for individual neurons, Figure $9 A$ plots the means and SEs of firing rates that were measured for pursuit at $20^{\circ} / \mathrm{s}$ and memory-guided saccades of $16^{\circ}$ in the preferred pursuit direction. Because time courses of these eye movements were so different, neuronal activity was measured for different time windows that were $400 \mathrm{~ms}$ in duration for pursuit and $150 \mathrm{~ms}$ for saccades. For each neuron, the time window was located within the $1000 \mathrm{~ms}$ period of target motion or the $600 \mathrm{~ms}$ period starting from $200 \mathrm{~ms}$ before saccade initiation and was located so as to obtain a maximal mean value. Time of the measurement interval varied from neuron to neuron but was the same for individual trials for each neuron. The plot in Figure $9 A$ indicates that actual firing rates for both types of eye movements are comparable, except for some neurons that showed modulation of activity during catch-up saccades (open symbols). However, because many neurons increased their activity during the delay period in the memory-guided saccade task (Fig. $8 A$ ), the comparison of firing rates could result from an interplay between preparatory activity and saccade activity. Therefore, modulation of neuronal activity
A

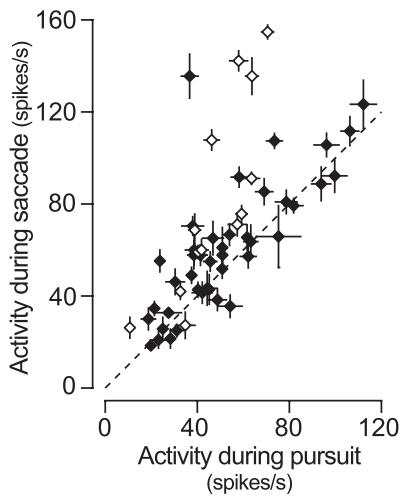

B

Figure 9. Comparison of activity during pursuit at $20^{\circ} / \mathrm{s}$ and memory-guided saccade of $16^{\circ}$. A, Each data point plots mean \pm SE firing rate measured for a $400 \mathrm{~ms}$ (pursuit) and a $150 \mathrm{~ms}$ (saccade) interval. Open symbols indicate data for 13 neurons that showed modulation of activity around the time of catch-up saccades. $\boldsymbol{B}$, Scatter plot comparing the magnitude of responses to different types of eye movements. For each neuron, baseline activity was subtracted from data shown in $\boldsymbol{A}$. Baseline activity was measured for $400 \mathrm{~ms}$ before target motion or $300-200$ ms before saccade initiation.

was also compared by subtracting baseline activity from actual firing rate for individual neurons (Fig. 9B). Baseline activity was measured for the $400 \mathrm{~ms}$ interval before target motion (pursuit) or for 300-200 ms before saccade initiation (saccade). Figure $9 B$ shows that many neurons exhibited a response to saccades, whereas 10 neurons (18\%) showed greater response to pursuit than saccades (one-tailed $t$ test, $p<0.05$ ). When ratio of the response magnitudes was computed for individual neurons (pursuit response divided by saccade response), the median of the value was 1.00, and the value was statistically less for neurons that responded to catch-up saccades than for the other neurons (Wilcoxon's rank-sum test, $p<0.01$ ).

\section{Activity of eye position-related neurons during pursuit}

A previous study showed that eye position signals in the central thalamus during fixation were affected by the direction of preceding saccades (Schlag-Rey and Schlag, 1984). Here, whether activity of eye position-related neurons was also affected by the direction and speed of ongoing smooth pursuit was investigated. Neuronal responses were examined by having monkeys track a moving target at different velocities within a limited range of eye positions that were $10^{\circ}$ from the center of the screen. Figure $10 \mathrm{~A}$ shows time courses of target position for five different pursuit tasks. A target spot appeared either at the center of the screen or at $10^{\circ}$ in the periphery and was then stepped $2-5^{\circ}$ and moved toward the position of fixation at $6,10,20$, or $30^{\circ} \%$ in the preferred direction of the neuron under study or moved at $20 \%$ in the opposite direction. The target crossed the initial fixation location $333,200,200$, and $167 \mathrm{~ms}$ after motion onset for a speed of 6,10 , 20 , and $30 \%$, respectively, and this step-ramp target motion effectively induced high gain pursuit and eliminated the occurrence of saccades during initiation of pursuit. The target stopped after 2000, 1200, 700, and $500 \mathrm{~ms}$ after its motion onset for target speed of $6,10,20$, and $30 \%$, respectively, and then remained visible for $>900 \mathrm{~ms}$ until the end of the trial. Figure $10 \mathrm{~B}$ shows the data of an eye position-related neuron that showed a preference for ipsilateral eye position. For pursuit in the direction of the preferred eye position, the neuron exhibited an increase in activity shortly after initiation of pursuit that had a latency of $\sim 100$ 

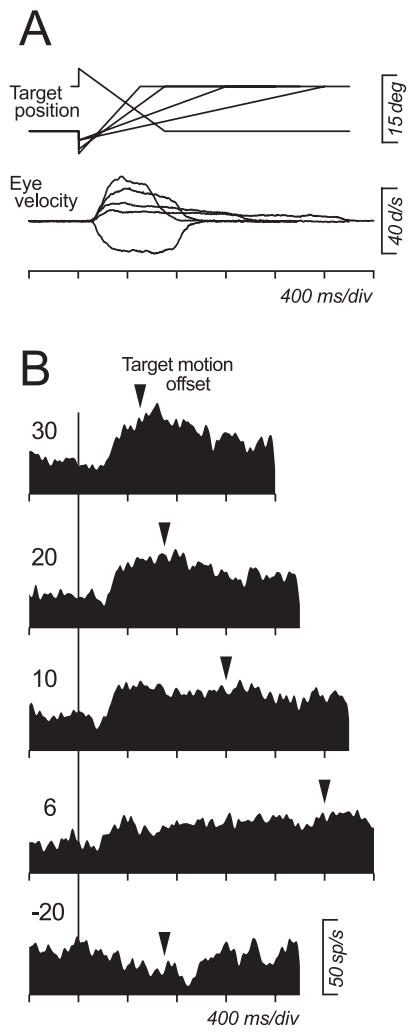

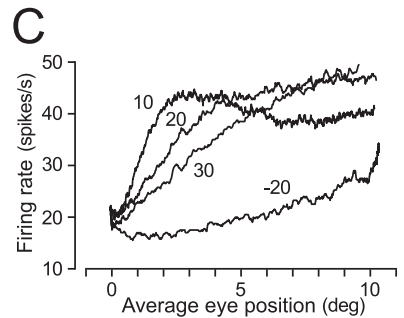

$\mathrm{D}$

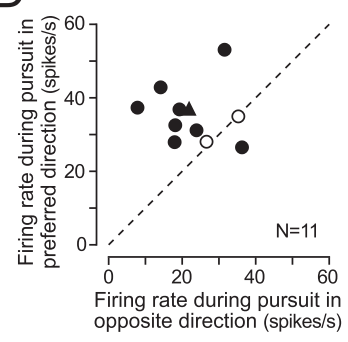

E

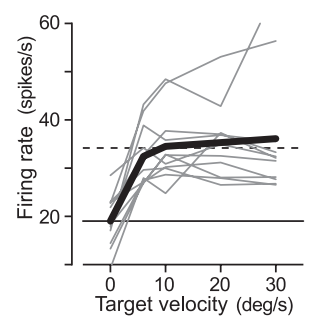

Figure 10. Responses of eye position-related neurons during smooth pursuit. $\boldsymbol{A}$, Time courses of target position and average eye velocity for the neuron shown in $\boldsymbol{B}$. The target stepped at the time of motion onset to eliminate saccades during pursuit initiation. $\boldsymbol{B}, \operatorname{Re}-$ sponses of an example neuron. Spike density profiles are aligned on target motion onset (vertical line). Numbers indicate target velocity. Note that the times of target motion offset (triangles) are different for different target speeds. $C$, Each trace plots mean firing rates measured for each 200 ms interval starting from $100 \mathrm{~ms}$ before to $100 \mathrm{~ms}$ after target motion as a function of mean horizontal eye position at the same measuring interval for the neuron shown in $\boldsymbol{B}$. Numbers indicate target velocity. D, Comparison of neuronal responses during pursuit at $20 \% \mathrm{~s}$ in opposite directions but within the same range of eye position. Filled symbols indicate neurons that showed statistically significant directional modulation during pursuit ( $t$ test, $p<0.05$ ). Triangle indicates data for the neuron shown in $\boldsymbol{B}$ and $\boldsymbol{C}$. $\boldsymbol{E}$, Responses to different target speeds in the preferred direction. Data connected with thin lines indicate individual neurons, and those connected with thick lines indicate population means. Data for zero target speed are the baseline activity measured during fixation before target motion onset. The horizontal solid and dashed lines indicate the population means of activity during fixation before and after target motion in the preferred direction, respectively.

ms. Firing rates grew more rapidly for faster target motion, and neuronal activity during the second fixation after target motion offset remained above baseline, consistent with eye position sensitivity. For target motion in the opposite direction, activity during initial fixation was rather high, and activity decreased during smooth pursuit. The slight increase in activity during central fixation after termination of target motion was likely attributed to occurrence of small saccades in the direction opposite to pursuit that compensated for the retinal error caused by a sudden offset of target motion.

To analyze the effects of smooth pursuit, firing rate was compared with eye position for each $200 \mathrm{~ms}$ interval starting from 100 $\mathrm{ms}$ before to $100 \mathrm{~ms}$ after target motion. Figure $10 \mathrm{C}$ plots the data of the neuron shown in Figure $10 B$ for four different target velocities. Although firing rate increased rather linearly as a function of eye position for target motion of $30 \%$, the relationship became nonlinear for other speeds. Because distortion of eye position signals during pursuit was greater for slower target motion, it could not be attributed to contamination of eye velocity or
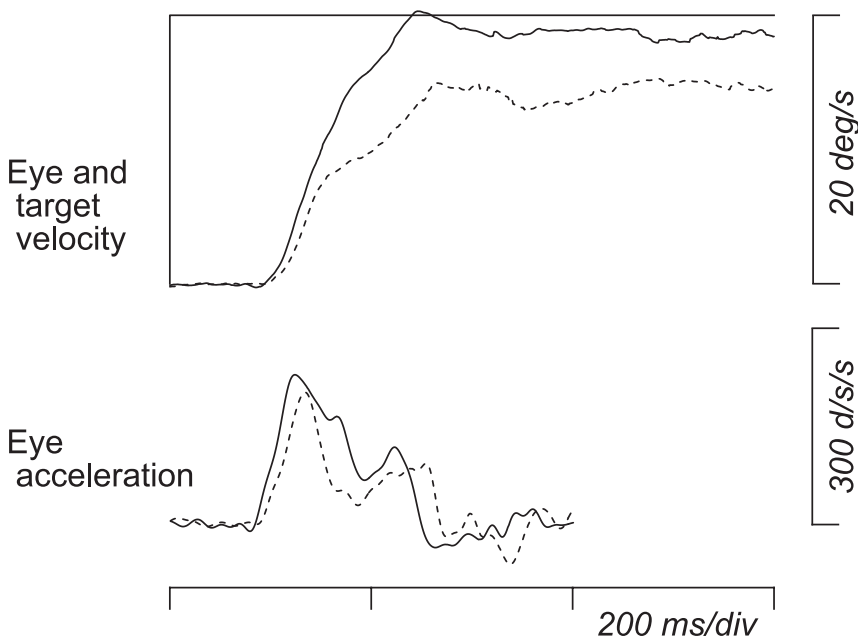

Figure 11. Effects of thalamic inactivation on smooth pursuit. Eye movement responses to ipsiversive target motion before (continuous trace) and after (broken trace) muscimol injection $(2 \mu \mathrm{l})$. Data of eye velocity are means of 48 and 53 desaccaded traces. Eye acceleration was computed from average eye velocity.

acceleration signals that must be greater for faster target motion. Conversely, neuronal activity during pursuit in the opposite direction was consistently less than that during pursuit in the preferred direction.

As an example shown in Figure 10C, most eye position-related neurons showed directional modulation for pursuit in a way that was expected from observations in a previous study (Schlag-Rey and Schlag, 1984): neuronal activity showed a trace of the direction of preceding eye movement, that is, the direction of ongoing pursuit. To quantify the effects of direction and speed of pursuit, neuronal activity was measured for periods of different lengths starting from $100 \mathrm{~ms}$ after target motion onset and ending at the time of target motion offset so that the range of eye position was the same for different target velocities. Figure $10 \mathrm{D}$ compares means of activity during pursuit at $20 \%$ s toward versus away from the preferred eye position for 11 eye position-related neurons. Although the eyes moved within the same range in both conditions, the magnitude of neuronal activity was greater for pursuit in the preferred direction than in the opposite direction for most neurons (73\%, 8 of 11). However, eye position-related neurons appeared to be insensitive to speed of target motion in the preferred direction. Figure $10 \mathrm{E}$ summarizes the activity during pursuit for different target speeds for individual neurons (thin lines) and for population means (thick solid line). Data are compared with means of activity during fixation before (solid horizontal line) and after (broken line) target motion that were measured for the $300 \mathrm{~ms}$ intervals immediately before target motion onset and that starting from $300 \mathrm{~ms}$ after target motion offset. Except for two neurons, neuronal responses did not correlate clearly with the speed of target motion in the preferred direction, indicating that eye position-related neurons could identify the direction of ongoing pursuit but not pursuit speed.

\section{Effects of inactivation on pursuit}

To examine whether pursuit-related signals in the thalamus are used for pursuit control, recording sites were inactivated reversibly by injecting a small amount of muscimol. Figure 11 shows time course of pursuit initiation for leftward target motion before (continuous traces) and after (broken traces) inactivation of the left thalamus. Effects of inactivation became evident shortly after 
A Pursuit initiation
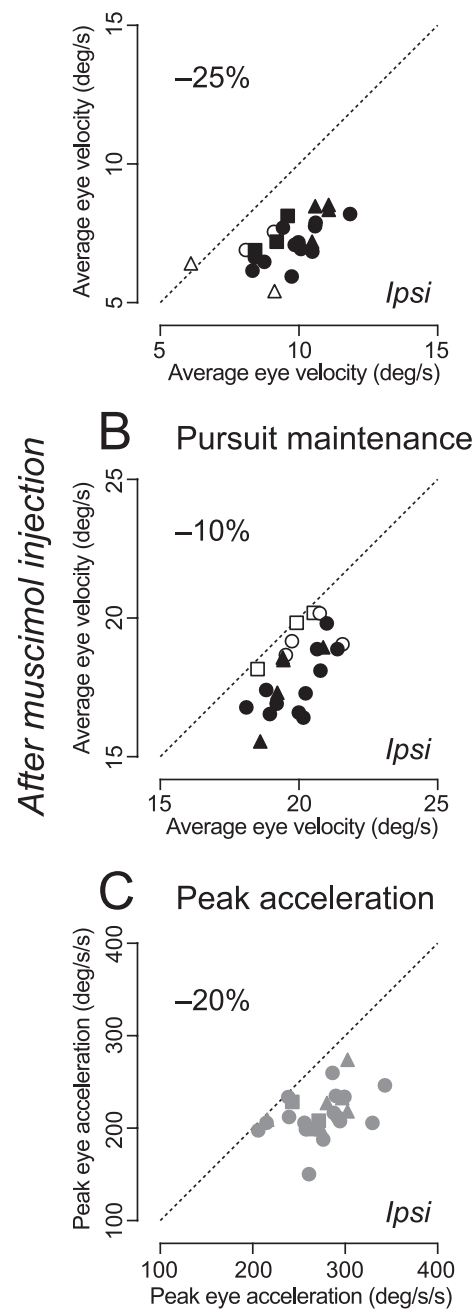

Before muscimol injection

Figure 12. Quantitative data of the effects of inactivation. $A$, Comparison of average pursuit velocity $100-200 \mathrm{~ms}$ after target motion onset. Data for injection sites that showed statistically significant effects (two-tailed $t$ test, $p<0.01$ ) are plotted as filled symbols. $\boldsymbol{B}$, Effects on eye velocity measured for $300-500 \mathrm{~ms}$ after target motion onset. C, Peak eye acceleration during $100-200 \mathrm{~ms}$ after target motion onset. In all panels, the different symbols indicate data from different animals. The number on each panel shows mean reduction of the value for 25 sites. The amount of muscimol ranged from 1.0 to $2.0 \mu$ land averaged $1.7 \pm 0.3 \mu \mathrm{l}$. Ipsi, Ipsiversive; Contra, contraversive.

pursuit initiation and persisted during maintenance of pursuit. To quantify these effects, the following three parameters were measured for horizontal pursuit at $20 \%$ in both directions: (1) average eye velocity $100-200 \mathrm{~ms}$ after target motion onset (initiation), (2) average eye velocity 300-500 ms after target motion onset (maintenance), and (3) peak eye acceleration during a period 100-200 ms after target motion onset (acceleration). These values were statistically different between before and after inactivation ( $t$ test, $p<0.05$ ) for pursuit in either or both directions for 25 of 39 sites tested. The locations of these effective sites in one monkey are shown in Figure 2 (gray circles). When saline was injected at 3 of the 25 sites, eye velocity was not altered during either initiation or maintenance of pursuit. Conversely, when effects on memory-guided saccades were tested for 21 of these 25 sites, latency of contraversive saccades was slightly but significantly (Wilcoxon's rank-sum test, $p<0.05$ ) lengthened for 13 sites. Figure 12 summarizes data of pursuit for 25 effective sites of
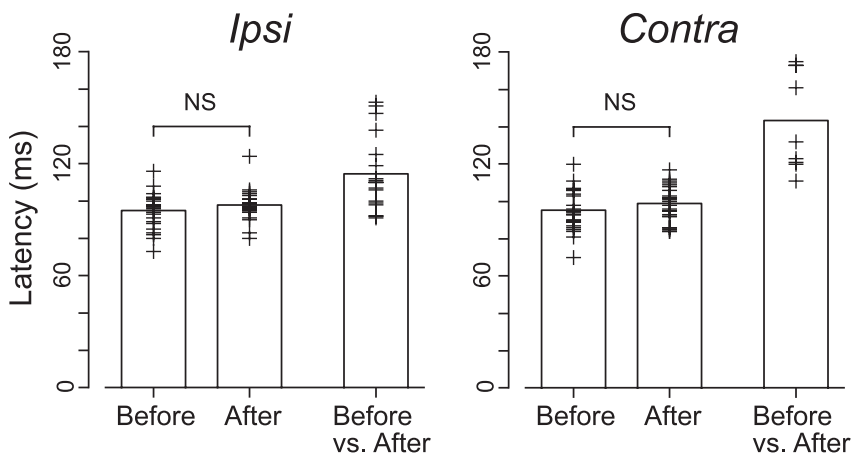

Figure 13. Comparisons between pursuit latency and onset of inactivation effects. In each panel, the left two columns plot the time when eye velocity after target motion was statistically different (two-tailed $t$ test, $p<0.01$ ) from baseline eye velocity measured before target motion. The right column plots the time when millisecond-by-millisecond eye velocity before and after inactivation were statistically different $(p<0.01)$ from each other for $>100 \mathrm{~ms}$. Cross symbols indicate data for individual injection sites. Latency of inactivation effects could be measured only for 18 and 9 sites for ipsiversive (Ipsi) and contraversive (Contra) pursuits, respectively.

three monkeys, comparing values before and after inactivation. The effect of inactivation was greater for ipsiversive than contraversive pursuit and was more evident during initiation than maintenance of pursuit. On average, eye velocity during pursuit initiation (Fig. 12A) became $75 \%$ (ranged from 58 to 105\%) and $92 \%(70-107 \%)$ of the control for ipsiversive and contraversive target motions, respectively. Peak eye acceleration (Fig. 12C) also reduced to $80 \%(59-96 \%)$ for ipsiversive pursuit but did not change greatly for contraversive pursuit ( $97 \%$ on average). Although the effect of inactivation on maintenance of pursuit was statistically significant ( $t$ test, $p<0.01$ ) (Fig. $12 B$, filled symbols) for many injection sites ( 21 and 14 of 25 sites for ipsiversive and contraversive pursuit, respectively), the amount of changes in average eye velocity was rather small. Eye velocity during maintenance of pursuit reduced to $90 \%(75-100 \%)$ and $94 \%(84-$ $103 \%$ ) of the control value for ipsiversive and contraversive target motions, respectively.

As shown in traces of pursuit velocity (Fig. 11), inactivation of the central thalamus did not alter pursuit latency. For each injection site and pursuit direction, pursuit latency was measured by comparing eye velocity for $100 \mathrm{~ms}$ before target motion onset (baseline) and the millisecond-by-millisecond eye velocity after target motion onset (see Materials and Methods). The two left columns in each panel in Figure 13 summarize pursuit latency before and after inactivation. For both directions, pursuit latency was not statistically different (NS, two-tailed $t$ test, $p>0.2$ ). Latency of inactivation effects was measured by comparing millisecond-by-millisecond pursuit velocity before and after inactivation (see Materials and Methods), and values are shown on the right column in each panel. On average, effects of inactivation appeared $114.5 \pm 20.3 \mathrm{~ms}(n=18)$ and $143.2 \pm 26.7 \mathrm{~ms}(n=9)$ after target motion onset for ipsiversive and contraversive pursuit, respectively. These values were $21.2 \pm 18.0$ and $45.7 \pm 31.2$ $\mathrm{ms}$ longer than latency of pursuit before inactivation for ipsiversive and contraversive pursuit, respectively.

\section{Discussion}

The present study provided direct evidence for thalamic involvement in the control of smooth pursuit eye movements. Many neurons in the ventrolateral thalamus exhibited sustained activity during pursuit in the preferred direction. Most of them discharged before or during initiation of pursuit, and their firing rates were proportional to the speed of target motion in the pre- 
ferred direction. Inactivation of these neurons reduced eye velocity during initiation and maintenance of pursuit, indicating that the central thalamus lies within pathways that regulate smooth pursuit eye movements.

\section{Properties of pursuit signals in the central thalamus}

So far, there has been no direct evidence showing that the central thalamus is involved in the control of smooth pursuit eye movements, although one study reported neurons that were inhibited during pursuit (Schlag and Schlag-Rey, 1984). Because this previous study showed that eye position signal in the central thalamus was affected by the direction of preceding eye movements, one might argue that modulation during pursuit was attributed to this hysteretic nature of eye position signals. Indeed, eye position-related neurons showed directional modulation during pursuit in a way that was expected from the previous study (Fig. 10). However, pursuit-related activity found in the present study was clearly different from directional modulation of eye positionrelated activity in several important ways. First, neurons lacked eye position sensitivity, showing no systematic changes in activity during fixation before and after eye movements in any directions. Second, many neurons with pursuit-related activity also exhibited a transient activity around the time of memory-guided saccades of $16^{\circ}$, whereas eye position-related neurons showed sustained activity as long as the monkey fixated a peripheral target in the preferred direction. Third, some neurons showed a weaker activity for pursuit in the direction opposite to the preferred direction, resulting in a wide distribution of the directional index (Fig. $3 B$ ). Finally, pursuit-related activity was sensitive to the speed of target motion in the preferred direction, whereas activity of eye position-related neurons was not. Because neurons continued firing when the tracking target was extinguished briefly, pursuit-related activity did not reflect motion of retinal target images. Instead, the activity was likely related to velocity of smooth pursuit.

Where do the pursuit signals come from? The present results showed that pursuit signals interacted with saccade signals in individual thalamic neurons. This result contrasts with previous anatomical (Tian and Lynch, 1997), physiological (Gottlieb et al., 1994), and functional imaging (Petit and Haxby, 1999; Rosano et al., 2002) data showing that pursuit and saccades are processed by distinct populations of cortical neurons. For example, only $\sim 10 \%$ of pursuit-related neurons in the FEF are active during saccades (Tanaka and Lisberger, 2002), although neurons in the ventrolateral thalamus project to the pursuit subregion in the FEF (Tian and Lynch, 1997). Conversely, it is known that pursuit and saccade signals are integrated in single neurons in the brainstem and the cerebellum, including the superior colliculus (Krauzlis et al., 1997), pontine reticular formation (Keller and Missal, 2003), interstitial nucleus of Cajal (Missal et al., 2000), vestibular nuclei (Chubb and Fuchs, 1982; Lisberger et al., 1994), fastigial nucleus (Fuchs et al., 1994), and cerebellar vermis (Suzuki and Keller, 1988) and flocculus (Lisberger and Fuchs, 1978). Because the paralaminar part of the thalamus receives inputs from all of these subcortical regions, interaction of both types of eye movements in thalamic neurons could be inherited from those subcortical neurons, although the proportion of pursuit neurons with saccade sensitivity was much greater in the thalamus than most of these subcortical regions. For example, a minority of pursuitrelated neurons in the thalamus that modulated their activity around the time of catch-up saccades may encode retinal position error during pursuit like neurons in the rostral superior colliculus (Krauzlis et al., 1997). Neurons with oblique preferred direction might receive pursuit signals from the vermis of the cerebellum through the fastigial nucleus in which interaction of both types of eye movement signals exists for 70 and $30 \%$ of pursuit neurons, respectively, and many respond to pursuit in oblique directions (vermis, Suzuki and Keller, 1988; fastigial nucleus, Fuchs et al., 1994).

Another possible source of pursuit signals in the central thalamus is the basal ganglia. Anatomically, the paralaminar regions of MD, VL, and VA nucleus of the thalamus receive strong projections from output nodes of the basal ganglia (Alexander et al., 1986). A recent study in Cebus monkeys showed that the pursuit subregion in the FEF received inputs from the pallidal-projecting part of VL and VA (Tian and Lynch, 1997). Furthermore, a recent imaging study showed significant increase in regional blood flow in the caudate nucleus during pursuit (O'Driscoll et al., 2000). In addition, it was observed that patients with Parkinson's disease showed abnormalities in pursuit (White et al., 1983), and unit recording experiments showed that a subset of neurons in the substantia nigra pars reticulata exhibited modulation in activity during pursuit (Pokorny and Basso, 2003). All of these studies support the hypothesis that the cortical-basal ganglia circuitry is involved in the control of smooth pursuit. Unfortunately, however, there are no available data to compare pursuit signals in the basal ganglia with the present results.

\section{Roles of the central thalamus in smooth pursuit eye movements}

Because the smooth pursuit system operates as a negative feedback control system, there must exist neuronal mechanisms that reconstruct target velocity during maintenance of pursuit by integrating signals of retinal image motion and ongoing pursuit velocity. Previous studies have incorporated positive feedback circuit(s) into the system to create target velocity signals in the absence of retinal image motion (Robinson et al., 1986; Krauzlis and Lisberger, 1994). There are several possible pathways that could account for this velocity memory of pursuit: recurrent networks between the cerebellum and the brainstem (Stone and Lisberger, 1990), networks within cerebral cortices (Tabata et al., 2002), and ascending thalamocortical pathways that link subcortical and cortical eye movement areas. The present study provided supporting evidence for the third pathway. Indeed, many thalamic neurons showed directional modulation during pursuit, and inactivation of these neurons decreased eye velocity during initiation and maintenance of pursuit. Consistent with the higher proportion of ipsiversive pursuit neurons, the effect of inactivation was greater for ipsiversive than contraversive pursuit. Thalamic inactivation did not alter pursuit latency but reduced eye velocity shortly after pursuit initiation, suggesting that the central thalamus lies within the internal feedback pathways.

However, effects of inactivation were only $25 \%$ for initiation and $10 \%$ for maintenance of pursuit. The small change in pursuit velocity might be attributed to the fact that pursuit-related neurons distributed sparsely within the central thalamus (Fig. 2). Local inactivation possibly removed pursuit signals that were carried by thalamic neurons only partially. Alternatively, velocity memory of pursuit might be implemented by multiple recurrent networks within the system, and thalamocortical pathways may play only a minor role in tracking condition in the present study. The idea of multiple recurrent circuits has already been suggested in several pursuit models (Robinson et al., 1986; Bennett and Barnes, 2004). Other studies have shown that the gain of feedback pathways is modifiable depending on the context of tracking conditions, including the presence of a stationary occluder (Churchland et al., 2003), practice (Madelain and Krauzlis, 2003), or predictability of target motion (Bennett and Barnes, 2004). Different 
feedback pathways might play roles in monitoring and regulating pursuit velocity in different conditions.

\section{References}

Alexander GE, DeLong MR, Strick PL (1986) Parallel organization of functionally segregated circuits linking basal ganglia and cortex. Annu Rev Neurosci 9:357-381.

Asanuma C, Thach WT, Jones EG (1983) Distribution of cerebellar terminations and their relation to other afferent terminations in the ventral lateral thalamic region of the monkey. Brain Res 286:237-265.

Baker JT, Harper TM, Snyder LH (2003) Spatial memory following shifts of gaze. I. Saccades to memorized world-fixed and gaze-fixed targets J Neurophysiol 89:2564-2576.

Bennett SJ, Barnes GR (2004) Predictive smooth ocular pursuit during the transient disappearance of a visual target. J Neurophysiol 92:578-590.

Chubb MC, Fuchs AF (1982) Contribution of y group of vestibular nuclei and dentate nucleus of cerebellum to generation of vertical smooth eye movements. J Neurophysiol 48:75-99.

Churchland MM, Chou IH, Lisberger SG (2003) Evidence for object permanence in the smooth-pursuit eye movements of monkeys. J Neurophysiol 90:2205-2218.

Fuchs AF, Robinson DA (1966) A method for measuring horizontal and vertical eye movement chronically in the monkey. J Appl Physiol 21:1068-1070.

Fuchs AF, Robinson FR, Straube A (1994) Participation of the caudal fastigial nucleus in smooth-pursuit eye movements. I. Neuronal activity. J Neurophysiol 72:2714-2728.

Gaymard B, Rivaud S, Pierrot-Deseilligny C (1994) Impairment of extraretinal eye position signals after central thalamic lesions in humans. Exp Brain Res 102:1-9.

Gottlieb JP, MacAvoy MG, Bruce CJ (1994) Neural responses related to smooth-pursuit eye movements and their correspondence with electrically elicited smooth eye movements in the primate frontal eye field. J Neurophysiol 72:1634-1653.

Huerta MF, Krubitzer LA, Kaas JH (1986) Frontal eye field as defined by intracortical microstimulation in squirrel monkeys, owl monkeys, and macaque monkeys. I. Subcortical connections. J Comp Neurol 253:415-439.

Kalil K (1981) Projections of the cerebellar and dorsal column nuclei upon the thalamus of the rhesus monkey. J Comp Neurol 195:25-50.

Keller EL, Missal M (2003) Shared brainstem pathways for saccades and smooth-pursuit eye movements. Ann NY Acad Sci 1004:29-39.

Krauzlis RJ, Lisberger SG (1994) A model of visually-guided smooth pursuit eye movements based on behavioral observations. J Comput Neurosci 1:265-283.

Krauzlis RJ, Basso MA, Wurtz RH (1997) Shared motor error for multiple eye movements. Science 276:1693-1695.

Lang W, Büttner-Ennever JA, Büttner U (1979) Vestibular projections to the monkey thalamus: an autoradiographic study. Brain Res 177:3-17.

Lisberger SG, Fuchs AF (1978) Role of primate flocculus during rapid behavioral modification of vestibuloocular reflex. I. Purkinje cell activity during visually guided horizontal smooth-pursuit eye movements and passive head rotation. J Neurophysiol 41:733-763.

Lisberger SG, Pavelko TA, Broussard DM (1994) Responses during eye movements of brain stem neurons that receive monosynaptic inhibition from the flocculus and ventral paraflocculus in monkeys. J Neurophysiol 72:909-927.

Lynch JC, Hoover JE, Strick PL (1994) Input to the primate frontal eye field from the substantia nigra, superior colliculus, and dentate nucleus demonstrated by transneuronal transport. Exp Brain Res 100:181-186.

Madelain L, Krauzlis RJ (2003) Effects of learning on smooth pursuit during transient disappearance of a visual target. J Neurophysiol 90:972-982.

Martin JH (1991) Autoradiographic estimation of the extent of reversible inactivation produced by microinjection of lidocaine and muscimol in the rat. Neurosci Lett 127:160-164.

Matelli M, Luppino G (1996) Thalamic input to mesial and superior area 6 in the macaque monkey. J Comp Neurol 372:59-87.

Missal M, de Brouwer S, Lefevre P, Olivier E (2000) Activity of mesencephalic vertical burst neurons during saccades and smooth pursuit. J Neurophysiol 83:2080-2092.

O’Driscoll GA, Wolff AV, Benkelfat C, Florencio PS, Lal S, Evans AC (2000) Functional neuroanatomy of smooth pursuit and predictive saccades. NeuroReport 11:1335-1340.
Petit L, Haxby JV (1999) Functional anatomy of pursuit eye movements in humans as revealed by fMRI. J Neurophysiol 82:463-471.

Pokorny J, Basso MA (2003) Participation of basal ganglia nucleus neurons in smooth pursuit eye movements. Soc Neural Control Mov Abstr 8:E-02.

Powell KD, Goldberg ME (1997) Remapping of visual responses in primate parietal cortex during smooth changes in gaze. Soc Neurosci Abstr 23:14.11.

Robinson DA, Gordon JL, Gordon SE (1986) A model of the smooth pursuit eye movement system. Biol Cybern 55:43-57.

Rosano C, Krisky CM, Welling JS, Eddy WF, Luna B, Thulborn KR, Sweeney JA (2002) Pursuit and saccadic eye movement subregions in human frontal eye field: a high-resolution fMRI investigation. Cereb Cortex 12:107-115.

Schlag J, Schlag-Rey M (1984) Visuomotor functions of central thalamus in monkey. II. Unit activity related to visual events, targeting, and fixation. J Neurophysiol 51:1175-1195.

Schlag J, Schlag-Rey M (1986) Role of the central thalamus in gaze control Prog Brain Res 64:191-201.

Schlag-Rey M, Schlag J (1984) Visuomotor functions of central thalamus in monkey. I. Unit activity related to spontaneous eye movements. J Neurophysiol 51:1149-1174.

Shook BL, Schlag-Rey M, Schlag J (1991) Primate supplementary eye field. II. Comparative aspects of connections with the thalamus, corpus striatum, and related forebrain nuclei. J Comp Neurol 307:562-583.

Sommer MA, Wurtz RH (2002) A pathway in primate brain for internal monitoring of movements. Science 296:1480-1482.

Sommer MA, Wurtz RH (2004a) What the brain stem tells the frontal cortex. I. Oculomotor signals sent from superior colliculus to frontal eye field via mediodorsal thalamus. J Neurophysiol 91:1381-1402.

Sommer MA, Wurtz RH (2004b) What the brain stem tells the frontal cortex. II. Role of the SC-MD-FEF pathway in corollary discharge. J Neurophysiol 91:1403-1423.

Stone LS, Lisberger SG (1990) Visual responses of Purkinje cells in the cerebellar flocculus during smooth-pursuit eye movements in monkeys. I. Simple spikes. J Neurophysiol 63:1241-1261.

Suzuki DA, Keller EL (1988) The role of the posterior vermis of monkey cerebellum in smooth-pursuit eye movement control. II. Target velocityrelated Purkinje cell activity. J Neurophysiol 59:19-40.

Tabata H, Yamamoto K, Kawato M (2002) Computational study on monkey VOR adaptation and smooth pursuit based on the parallel controlpathway theory. J Neurophysiol 87:2176-2189.

Tanaka M (2003) Contribution of signals downstream from adaptation to saccade programming. J Neurophysiol 90:2080-2086.

Tanaka M (2004) Smooth pursuit signals in the central thalamus of monkeys. Soc Neurosci Abstr 30:186.2.

Tanaka M, Lisberger SG (2002) Role of arcuate frontal cortex of monkeys in smooth pursuit eye movements. I. Basic response properties to retinal image motion and position. J Neurophysiol 87:2684-2699.

Tanibuchi I, Goldman-Rakic PS (2003) Dissociation of spatial-, object-, and sound-coding neurons in the mediodorsal nucleus of the primate thalamus. J Neurophysiol 89:1067-1077.

Tanibuchi I, Goldman-Rakic PS (2005) Comparison of oculomotor neuronal activity in paralaminar and mediodorsal thalamus in the rhesus monkey. J Neurophysiol 93:614-619.

Tian JR, Lynch JC (1997) Subcortical input to the smooth and saccadic eye movement subregions of the frontal eye field in Cebus monkey. J Neurosci 17:9233-9247.

Watanabe Y, Funahashi S (2004) Neuronal activity throughout the primate mediodorsal nucleus of the thalamus during oculomotor delayed-responses. I. Cue-, delay-, and response-period activity. J Neurophysiol 92:1756-1769.

White OB, Saint-Cyr JA, Tomlinson RD, Sharpe JA (1983) Ocular motor deficits in Parkinson's disease. II. Control of the saccadic and smooth pursuit systems. Brain 106:571-587.

Wyder MT, Massoglia DP, Stanford TR (2003) Quantitative assessment of the timing and tuning of visual-related, saccade-related, and delay period activity in primate central thalamus. J Neurophysiol 90:2029-2052.

Wyder MT, Massoglia DP, Stanford TR (2004) Contextual modulation of central thalamic delay-period activity: representation of visual and saccadic goals. J Neurophysiol 91:2628-2648.

Zivotofsky AZ, Rottach KG, Averbuch-Heller L, Kori AA, Thomas CW, Dell'Osso LF, Leigh RJ (1996) Saccades to remembered targets: the effects of smooth pursuit and illusory stimulus motion. J Neurophysiol 76:3617-3632. 